\title{
Particular electrocardiographic phenotypes in acute coronary syndromes
}

\author{
Anamaria Avram ${ }^{1,2}$, Radu Brezeanu' ${ }^{2}$, Alexandru lancu'2 , Valentin Chioncel ${ }^{1,2}$, \\ Catalina Andrei ${ }^{1,2}$, Crina Sinescu ${ }^{1,2}$ \\ 1"Carol Davila" University of Medicine and Pharmacy, Bucharest, Romania \\ 2"Bagdasar-Arseni" Emergency Clinical Hospital, Bucharest, Romania
}

\begin{abstract}
Despite continuous efforts in early recognition and timely management, acute coronary syndromes (ACS) continue to be the most common cause of death worldwide.

The electrocardiogram (ECG) is the fastest, repeatable and most accesible instrument with diagnostic value, prognostic significance and therapeutic implications.

Based on the ECG, ACS are divided into ST segment elevation myocardial infarction (STEMI) and non-ST segment elevation ACS (NSTEACS). Current guidelines recommend emergency reperfusion theraphy only in patients with STEMI. Conventional criteria for the diagnosis of STEMI exclude the patients with atypical ECG findings, correlated with an increased risk of transmural myocardial infarction and considered STEMI equivalents. These particular ECG phenotypes are: new or presumably new bundle branch block, ST segment elevation in aVR, isolated posterior myocardial infarction, de Winter T waves, Wellens syndrome and ischaemia induced Brugada phenocopy.

Rapid risk stratification in patients with NSTEACS is crucial for adequate management. The particular ECG phenotypes discussed herein proove the need to redefine the signs of the present or iminent coronary artery occlusion, especially the left anterior descending (LAD) artery, because many patient may benefit from early invasive treatment instead of conservative pharmacological treatment.
\end{abstract}

Keywords: acute coronary syndromes, risk stratification, emergency reperfusion theraphy, STEMI equivalents

\section{INTRODUCTION}

Despite continuous efforts in early recognition and timely management, acute coronary syndromes (ACS) continue to be the most common cause of death worldwide [1]. The conventional 12-lead ECG is the golden standard as the first diagnostic test [2]. Fifty years ago Eugene Braunwald issued the concept „time is muscle", highlighting that the longer the ischaemic time, the more muscle is lost [3]. Thus, the correct interpretation of the ECG is essential for rapid therapeutic interventions to rescue the myocardium at risk or to limit the infarcted area.

\section{DISCUSSION}

Based on the ECG, ACS divide into ST-segment elevation myocardial infarction (STEMI) and nonST-segment elevation ACS (NSTEACS). Current guidelines recommend emergency reperfusion theraphy only in patients with STEMI [4]. Conventional criteria for the diagnosis of STEMI exclude the patients with atypical ECG findings, although they are correlated with an increased risk of transmural myocardial infarction. These atypical ECG presentations and considered life-threatening STEMI equivalents and should prompt consideration of urgent cardiac catheterization. The particular ECG phenotypes discussed herein are: new or presumably new bundle branch block, ST segment elevation in aVR, isolated posterior myocardial infarction (MI), de Winter $\mathrm{T}$ waves, Wellens syndrome and ischaemia-induced Brugada phenocopy.

\section{New or presumably new bundle branch block}

The third universal definition of myocardial infarction offers diagnostic value to the rise and/or 
fall of the myocardial necrosis biomarkers along with new or presumed new ST-T changes or new left bundle branch block (LBBB) [5]. The fourth universal definition of MI recognises the diagnostic value of the new non-rate-related right bundle branch block as well [6].

a) Left bundle branch block

Left bundle branch block represents a challenge in the evaluation of the patient with chest pain because the standard criteria for ST segment deviation are unusable due to secondary ST-T changes. In order to overcome this pitfall, a number of algorithms have been elaborated over time [7]. In 1996, Sgarbossa issued the Sgarbossa criteria in order to assist the diagnosis of MI in the presence of LBBB (or a ventricular paced rhythm):

- ST segment elevation $\geq 1 \mathrm{~mm}$ in a lead with a positive QRS complex (concordance) - 5 points;

- concordant ST segment depression $\geq 1 \mathrm{~mm}$ in leads $\mathrm{V} 1, \mathrm{~V} 2$, or $\mathrm{V} 3-3$ points;

- ST segment elevation $\geq 5 \mathrm{~mm}$ in a lead with a negative QRS complex (discordance) -2 points [8].

When using Sgarbossa criteria, a score $\geq 3$ points has $90 \%$ specificity for MI [8].

In 2012 Smith et al. modified the original Sgarbossa criteria replacing the last criterion, which uses an absolute of $5 \mathrm{~mm}$ ST segment deviation, with an ST/S ratio of equal to or more than 0.2 in the presence of at least $2 \mathrm{~mm}$ of ST segment elevation. Modified Sgarbossa criteria improve the predictive power for acute coronary occlusion [9].

The 2017 European Society of Cardiology (ESC) guidelines for the management of STEMI list the criteria that can be used to improve the diagnostic accuracy of myocardial infarction in the presence of LBBB:

- concordant ST segment elevation $\geq 1 \mathrm{~mm}$ in leads with a positive QRS complex;

- concordant ST segment depression $\geq 1 \mathrm{~mm}$ in V1-V3;

- discordant ST segment elevation $\geq 5 \mathrm{~mm}$ in leads with a negative QRS complex [4].

Patients with a clinical suspicion of myocardial ischaemia and $\mathrm{LBBB}$ should be managed similar to STEMI patients, regardless of weather the LBBB is previously known [4].

Figure 1 presents the ECG of a patient with ACS and new intermitent LBBB. The tracing captures the exact moment that $\mathrm{LBBB}$ resolves, unmasking the ischaemic abnormalities underneath (poor $\mathrm{R}$ wave progression in leads $\mathrm{V} 1-\mathrm{V} 3$, biphasic $+/-\mathrm{T}$ waves V1-V4).

b) Right bundle branch block

The diagnosis of transmural myocardial ischaemia may be difficult in the presence of RBBB. In a

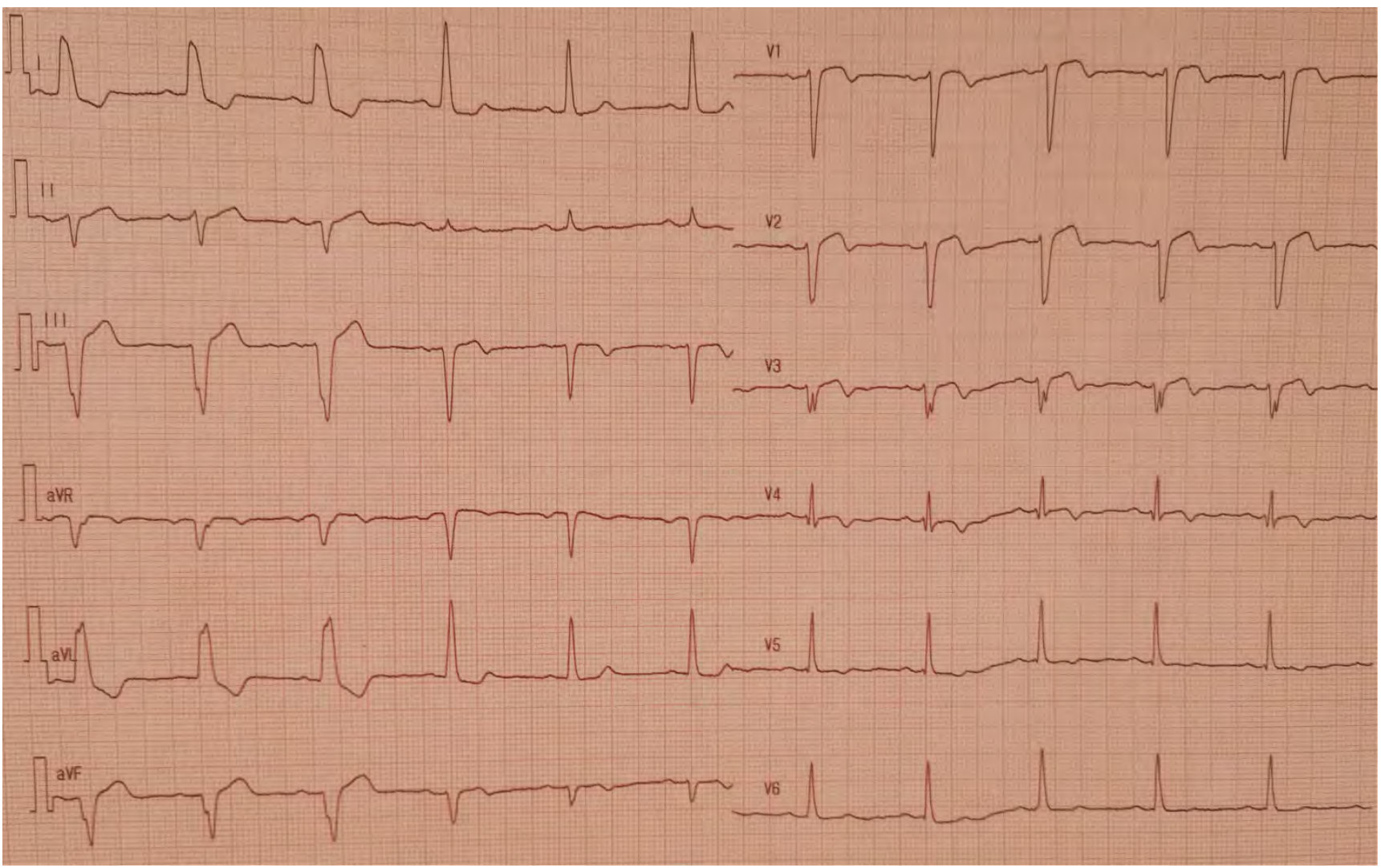

FIGURE 1. ECG in a patient with ACS and new intermitent $L B B B$ 
study $53 \%$ of the patients with MI and RBBB had ST segment elevation, while the rest had no ST segment deviation [10]. Patients with MI and new or presumably new RBBB have an advanced Killip class on admission (15.4\% present with cardiogenic shock) and a poor prognosis since in-hospital mortality is highest of all ECG presentations of MI, especially when associated with left anterior or left posterior fascicular block (18.8\% compared with $13.2 \%$ in patients with new or presumably new LBBB, $10,1 \%$ in patients with old LBBB and $6.4 \%$ in patients with old RBBB) [10]. As a consequence, the 2017 ESC guidelines for the management of STEMI included for the first time the patients with MI and new or presumably new RBBB as an indication for reperfusion theraphy, regardless of the presence or absence of ST segment deviation [4].

\section{ST segment elevation in aVR}

The presence of ST segment elevation in aVR and/or $\mathrm{V}_{1}$ together with $\mathrm{ST}$ segment depression $\geq 1$ $\mathrm{mm}$ in 8 or more surface leads (infero-lateral territory) suggests iminent left main (LM) occlusion, left main equivalents (significant disease of the LAD and the circumflex artery), LAD occlusion, severe three-vessel disease or severe diffuse transmural ischaemia [11]. The absence of ST segment elevation in aVR practically excludes significant disease of the LM. Subocclusion or complete occlusion of the LM is usually associated with hae- modynamic compromise (cardiogenic shock) and the development of ST segment elevation in the antero-lateral leads and has a high mortality rate [12]. Thus, rapid recognition of this atypical ECG presentation of MI should prompt a primary percutaneous coronary intervention strategy for prognostic improvement.

Figure 2 depicts an ECG showing concomitant ST-segment elevation in aVR and new RBBB in a patient with angiographically proven LM subocclusion.

\section{Isolated posterior myocardial infarction}

Posterior MI represents $15-20 \%$ of STEMI patients, usually in association with inferior or lateral territory, while isolated posterior MI represent only $5 \%$ of STEMI [13]. Posterior MI involves the basal part of the left ventricle (LV), usually supplied by the circumflex artery. The 12-lead ECG findings in posterior MI include (figure 3 ):

- horizontal, ascending or descending ST segment depression in V1-V3;

- proeminent $\mathrm{R}$ waves $>30 \mathrm{~ms}$ in $\mathrm{V} 1-\mathrm{V} 3$ (or equal voltage of the $\mathrm{R}$ and $\mathrm{S}$ waves in $\mathrm{V} 1$ );

- $\mathrm{R} / \mathrm{S}$ ratio $>1$ in $\mathrm{V} 2$ [14].

Due to the absence of ST-segment elevation on the 12-lead ECG, the diagnosis is frequently missed. The ECG findings previously described represent the indirect, ,mirror" image of the posterior territory. The direct image showing ST seg-

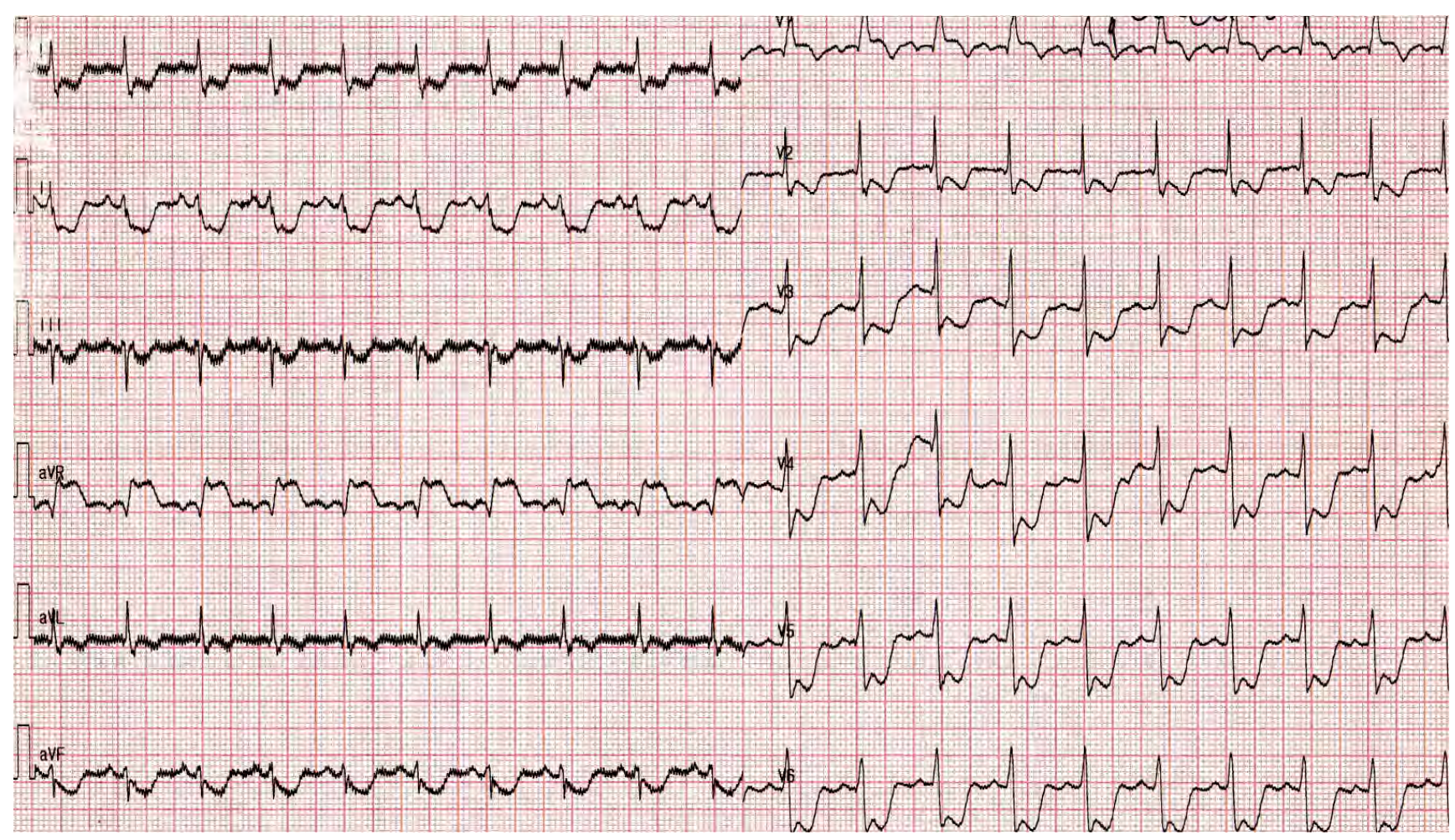

FIGURE 2. ECG in a patient with LM subocclusion: concomitant ST-segment elevation in aVR and new RBBB 


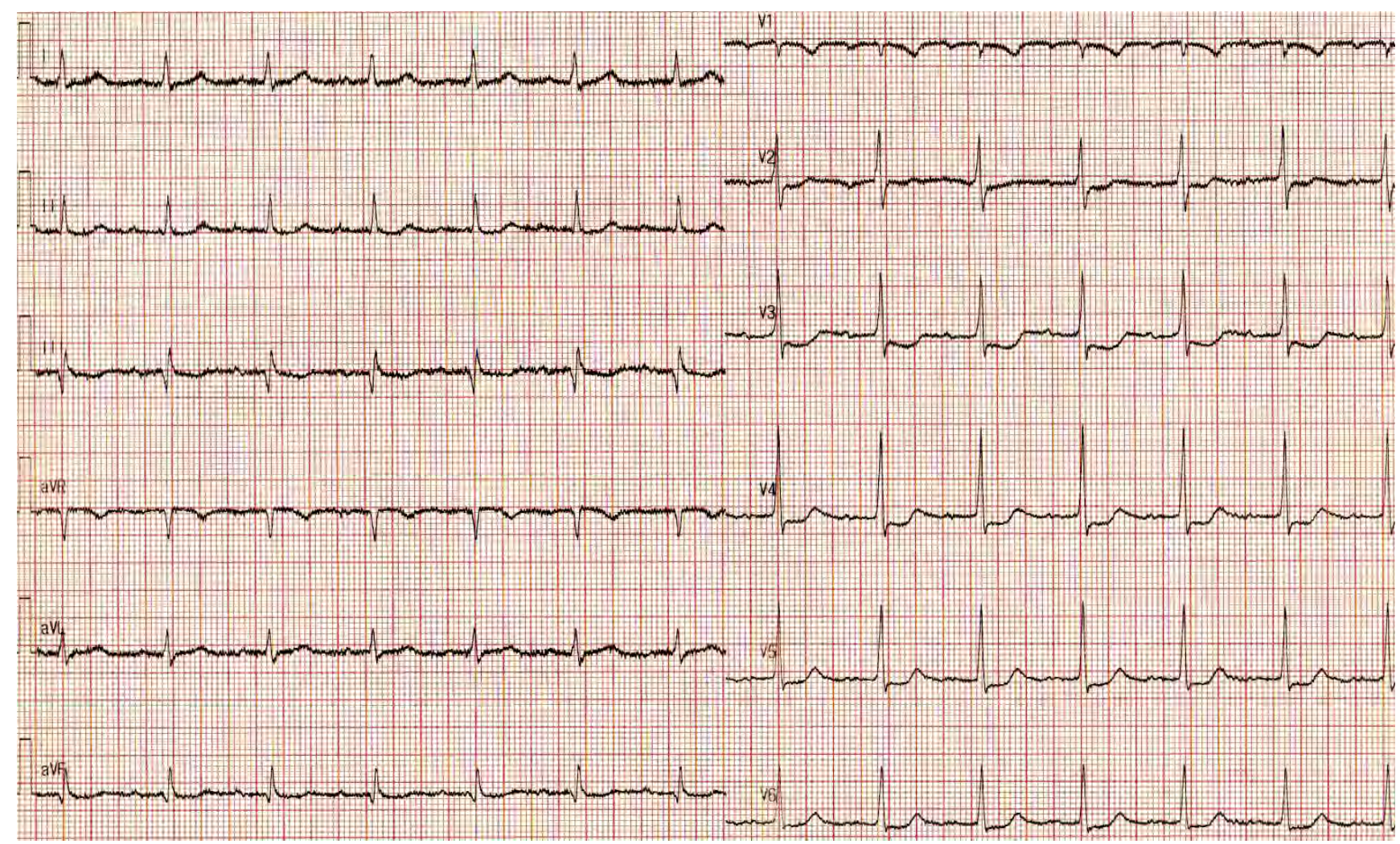

FIGURE 3. ECG fulfilling the criteria for posterior myocardial infarction

ment elevation is seen in additional posterior leads (V7-V9). The diagnostic criteria for posterior STEMI accepted by the ESC guidelines for the management of STEMI are:

- ST segment elevation $\geq 0.5 \mathrm{~mm}$ in V7-V9 ( $\geq 1 \mathrm{~mm}$ in men $<40$ years);

- ST segment depression $\geq 0.5 \mathrm{~mm}$ in V1-V3 [4].

As a rule of the thumb, in patients with clinical suspicion of myocardial ischaemia and initial nondiagnostic ECG, the tracing should be repeated in order to unmask dynamic ST segment deviation, and additional posterior $\left(\mathrm{V}_{7}-\mathrm{V}_{9}\right)$ or right precordial $\left(\mathrm{V}_{3} \mathrm{R}\right.$ and $\left.\mathrm{V}_{4} \mathrm{R}\right)$ leads should be used in patients with high suspicion of posterior MI or inferior MI with right ventricular involvement, respectively [4].

\section{De Winter T waves}

In 2008, Robert J. Winter and J.J. Wellens analized the data in their percutaneous coronary interventions database and described an eponymous sign (de Winter T waves) associated with proximal LAD occlusion in the absence of ST segment elevation [15]. The diagnostic elements of de Winter pattern are (figure 4):

- ascending ST segment depression $>1 \mathrm{~mm}$ in leads $\mathrm{V}_{1}-\mathrm{V}_{6}$;
- positive symetrical tall $\mathrm{T}$ waves taking off under the isoelectric line in leads $\mathrm{V}_{1}-\mathrm{V}_{6}[16]$.

The duration of the QRS complexes may be normal or slightly increased. Most of the patients present ST segment elevation in lead aVR. In some cases there is poor $\mathrm{R}$ wave progression in precordial leads [15]. Unlike hyperacute $\mathrm{T}$ waves occuring within minutes after acute coronary occlusion and rapidly evolving towards ST segment elevation, de Winter $\mathrm{T}$ waves are not transient, instead they persist on subsequent tracings [17]. The prevalence of this pattern is about $2 \%$ of the patients with MI and angiographically proven LAD occlusion, both in the initial study of de Winter and Wellens in 2008 (30/1,532 pts) [15] and in Verouden`s study in 2009 $(35 / 1,890 \mathrm{pts})[18]$.

\section{Wellens syndrome}

Gerson et al. first described the occurence of inverted terminal $\mathrm{T}$ waves (assigned as $\mathrm{U}$ wave inversion) in precordial leads in patients with LAD ischaemia. In 1979 they reported this abnormality during exercise [19] and in 1980 they evaluated the same abnormality at rest [20]. In both situations the patients with this ECG finding had evedence of LAD or left main ischaemia.

In 1980 de Zwaan, Wellens et al. described a similar ECG pattern in patients with unstable angi- 


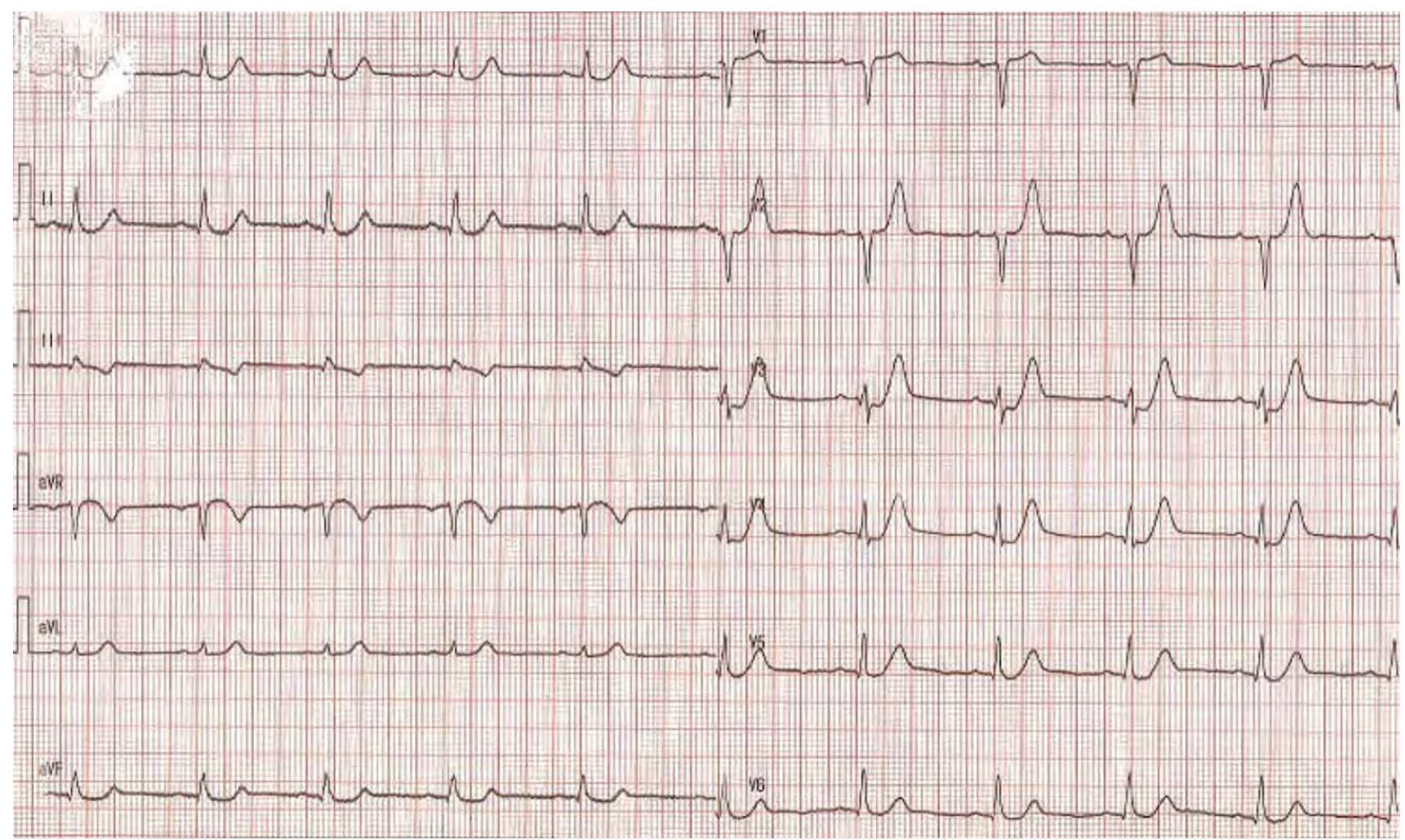

FIGURE 4. ECG showing de Winter pattern

na, thereafter reffered to as Wellens syndrome. The authors concluded that patients with such ECG findings represent a subgroup with unfavorable clinical course if treated conservatively. At that time in most hospitals the usual approach of patients with unstable angina was pharmacological management with nitroglycerin and betablocade. Only patients not responding to this type of management within a few days were considered suited for coronary angiography. Of the 26 patients admitted for unstable angina who showed the typical ST-T pattern, only 10 were reffered for coronary angiography and all of them had $\mathrm{a} \geq 90 \%$ stenosis of the LAD. Of the remaining 16 patients managed conservatively, $12(75 \%)$ developed an extensive anterior wall MI [21].

Wellens syndrome is correlated with a critical LAD stenosis due to acute plaque rupture resulting in temporary obstruction of the blood flow, followed by spontaneous reperfusion before extensive MI develops [22]. Thus, Wellens syndrome is considered a premyocardial infarction stage.

According to the initial description, the typical ECG findings consist of an isoelectric or minimally elevated $(1 \mathrm{~mm})$ takeoff of the ST segment from the QRS complex, a concave or straight ST segment passing into a negative $T$ wave at an angle of $60^{\circ}$ to $90^{\circ}$, and a symmetrically inverted $\mathrm{T}$ wave [21]. The authors recognised two distinct morphologic types:
- type A, less frequent: represents $25 \%$ of the cases and is characterised by isoelectric or minimally elevated $(1 \mathrm{~mm})$ takeoff of the ST segment (J point elevation), a concave or straight ST segment passing into the first part of the $\mathrm{T}$ wave at an angle of approximately $135^{\circ}$, followed by $\mathrm{T}$ wave inversion (figure 5 ).

- type B, the common type: represents $75 \%$ of the cases and is characterised by a takeoff of the ST segment from the QRS complex below the isoelectric line (J point depression), a convex ST segment passing into a negative $\mathrm{T}$ wave at an angle of about $120^{\circ}$, followed by a deep symmetrically inverted T wave (figure 6) [21].

The diagnostic criteria for Wellens syndrome have been introduced by Rinehardt $\mathrm{J}$ et al. in 2002:

- symmetric and deeply inverted $\mathrm{T}$ waves in leads V2 and V3 (occasionally in leads V1, $\mathrm{V} 4, \mathrm{~V} 5$, and V6);

or

- biphasic (+/-) T waves in leads V2 and V3; plus

- isoelectric or minimally elevated $(<1 \mathrm{~mm})$ ST segment;

- no precordial Q waves;

- ECG pattern present in pain-free state;

- history of recent angina;

- normal or slightly elevated serum cardiac necrosis markers [23]. 


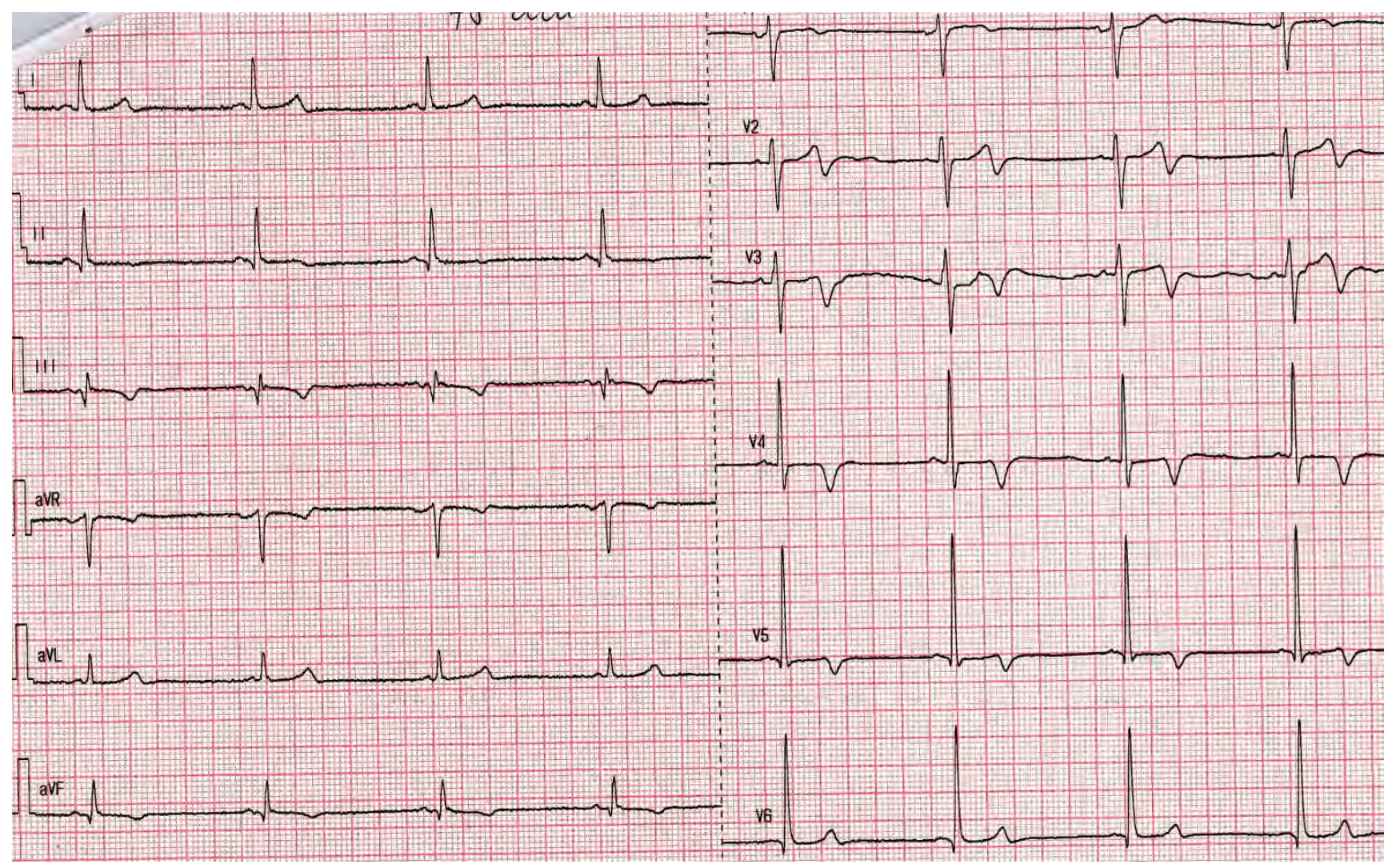

FIGURE 5. Type A Wellens syndrome

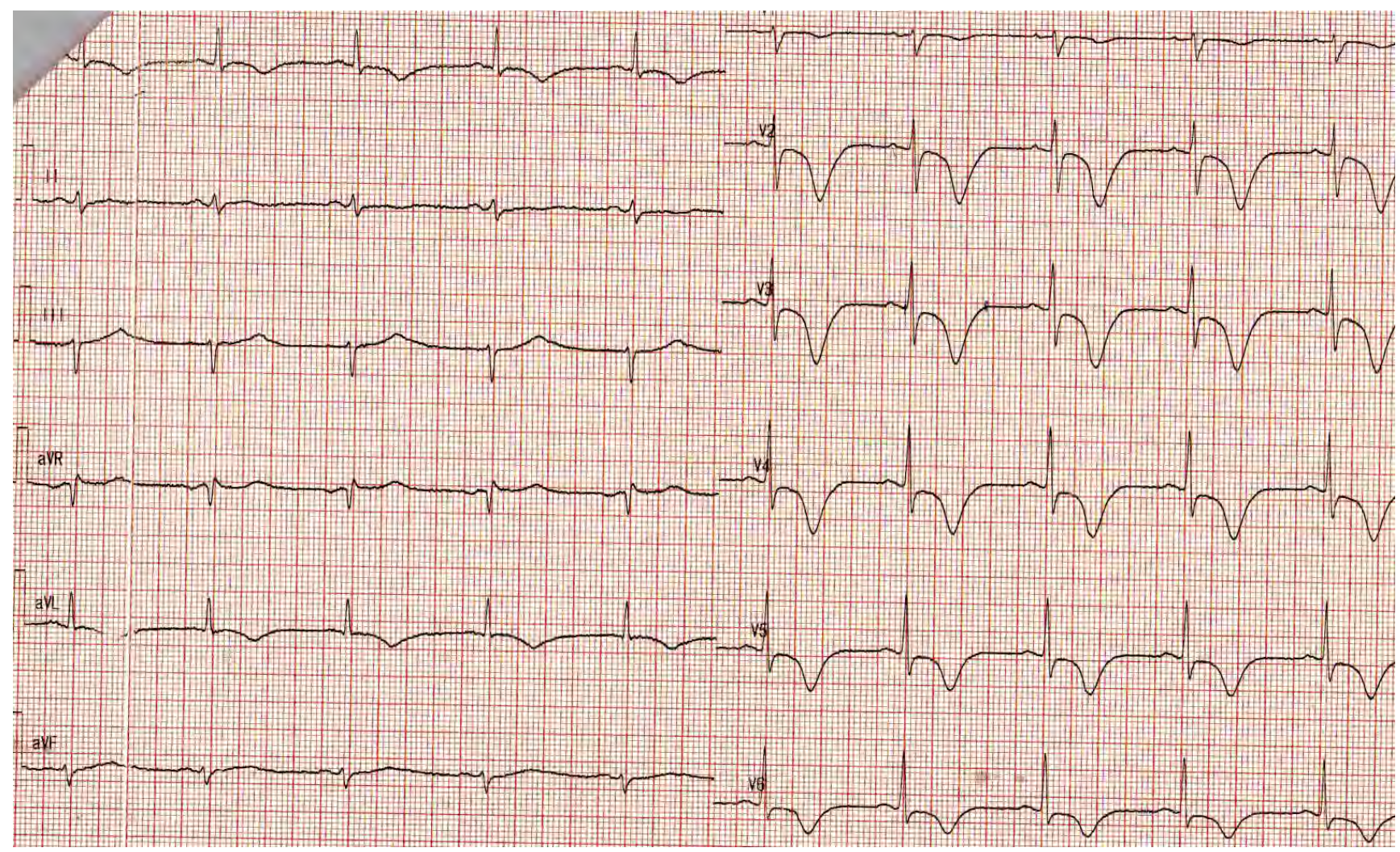

FIGURE 6. Type B Wellens syndrome

By the moment the prognostic value of Wellens sign for LAD critical stenosis in patients with unstable angina was recognised, sensitive cardiac biomarkers (high sensitivity troponins) were not available. Thus, we can assume that some of the patients with unstable angina would have had troponin elevation and would have been diagnosed with NSTEMI if cardiac troponin had been available. 
The prevalence of Wellens sign and its predictive value for LAD culprit lesion have not been clarified in a contemporary NSTEMI cohort. In 2019 , Kobayashi et al. published a study of 274 patients with NSTEMI, of whom 24 (8.8\%) had Wellens sign. Patients with Wellens sign were more likely to have LAD culprit lesion, compared to those without it $(66.7 \%$ vs. $19.6 \%, \mathrm{P}<0.001)$. Sensitivity and specificity of Wellens' sign to predict LAD critical stenosis were $24.6 \%$ and $96.2 \%$, respectively. Notably, despite its high specificity of Wellens' sign for LAD lesion, it was also observed in patients with non-LAD culprit lesions and non-obstructive coronary artery disease [24].

\section{Ischaemia-induced Brugada phenocopy}

Brugada syndrome has been described for the first time in 1992 by the brothers Pedro and Josep Brugada and is considered a primary electrical disease (channelopathy), in the absence of significant structural heart disease [25]. Brugada syndrome has the potential to determine lethal ventricular arrhythmias (polymorphic ventricular tachycardia or ventricular fibrillation) in young adults. The disease has genetic determinism with autosomal dominant transmission and incomplete penetrance and is caused by a series of mutations in the genes encoding subunits of the transmembranar ionic channels, resulting in loss of function of sodium (INa) or calcium (ICa) channels or gain of function of transient outward (Ito) or ATP-sensitive (IK-ATP) potassium currents [26]. The abnormalities are more proeminent in the epicardium of the right ventricular outflow tract and consist of the alteration of the local action potential, with resultant transmural (endo-epicardic) voltage gradient during repolarisation. This transmural dispersion of repolarization may precipitate the development of phase 2 reentry (local reexcitation), with the occurence of short coupled premature ventricular beats which can degenerate into polymorphic ventricular tachycardia or ventricular fibrillation [27].

Brugada syndrome is characterized by a particular type of ST segment elevation in leads $\mathrm{V}_{1}-\mathrm{V}_{3}$, better seen by placing the leads $\mathrm{V}_{1}$ and $\mathrm{V}_{2}$ in the second or the third intercostal space [26]. There are three ECG types, but only spontaneous type 1 is considered diagnostic and consists of $\mathrm{J}$ point elevation $\geq 0.2 \mathrm{mV}(2 \mathrm{~mm})$ imitating incomplete right branch bundle block (RBBB) followed by coved ST segment elevation and negative $T$ waves in at least one right precordial lead $\left(\mathrm{V}_{1}-\mathrm{V}_{3}\right)$ positioned in the $4^{\text {th }}, 3^{\text {rd }}$ or $2^{\text {nd }}$ intercostal space. The other types may be converted into type 1 using challenge tests (pharmacologic test, during a febrile episode or after the ingestion of a large meal). Recently, the diagnostic value of a positive challenge test has been argued and it should be considered as probabilistic rather than binary in nature [26].

Type 1 Brugada has dynamic character as it is modulated and unmasked by a series of physiologic, metabolic, pharmacologic or hormonal factors. These factors may also act as precipitants of malignant ventricular arrhythmias [25].

Not all provoked Brugada patterns reflect genetically Brugada syndrome. Acquired Brugada syndrome is called phenocopy. A phenocopy presents undistinguishable ECG pattern, but in the absence of any genetic abnormality, is associated with a specific clinical condition and reverts after removing the trigger, and familial history, personal history and challenge tests are negative [28]. These factors include myocardial ischaemia, pulmonary embolism, metabolic conditions (hyponatremia, hyperkalemia, hypokalemia, hypophosphatemia), mechanical compression (pectus excavatum, mediastinal compression), myocardial and pericardial disease [25]. Myocardial ischaemia presenting with Brugada pattern may be a modulator of genetically Brugada syndrome or a phenocopy. The largest series of patients with ischaemia-induced Brugada phenocopy consists of 17 cases from the International Registry of Brugada Phenocopies. In all cases Brugada pattern reverted in 2 min to 5 hours after the correction of ischaemia, confirming its role as a trigger. Pharmacological provocation tests were performed in $7 / 17 \mathrm{pts}(41 \%)$ and none was positive. No patient had malignant ventricular arrhythmias in the acute phase or during follow-up [29].

Figure 7 shows the ECGs of a patient admitted to our hospital for chest pain. At presentation the ECG revealed Brugada type 1 pattern (A), then, when the chest pain resolved, the ECG showed type A Wellens pattern (B). Coronary angiography performed the same day revealed $90 \%$ stenosis of the proximal LAD and it was treated succesfully by angioplasty with implantation of one drug eluting stent.

\section{CONCLUSIONS}

Non-ST-segment elevation ACS encompass a heterogenous grup in terms of severity of the coronary disease and, as a consequence, present major differences in prognosis. Rapid risk stratification in patients with NSTEACS is crucial for adopting the 


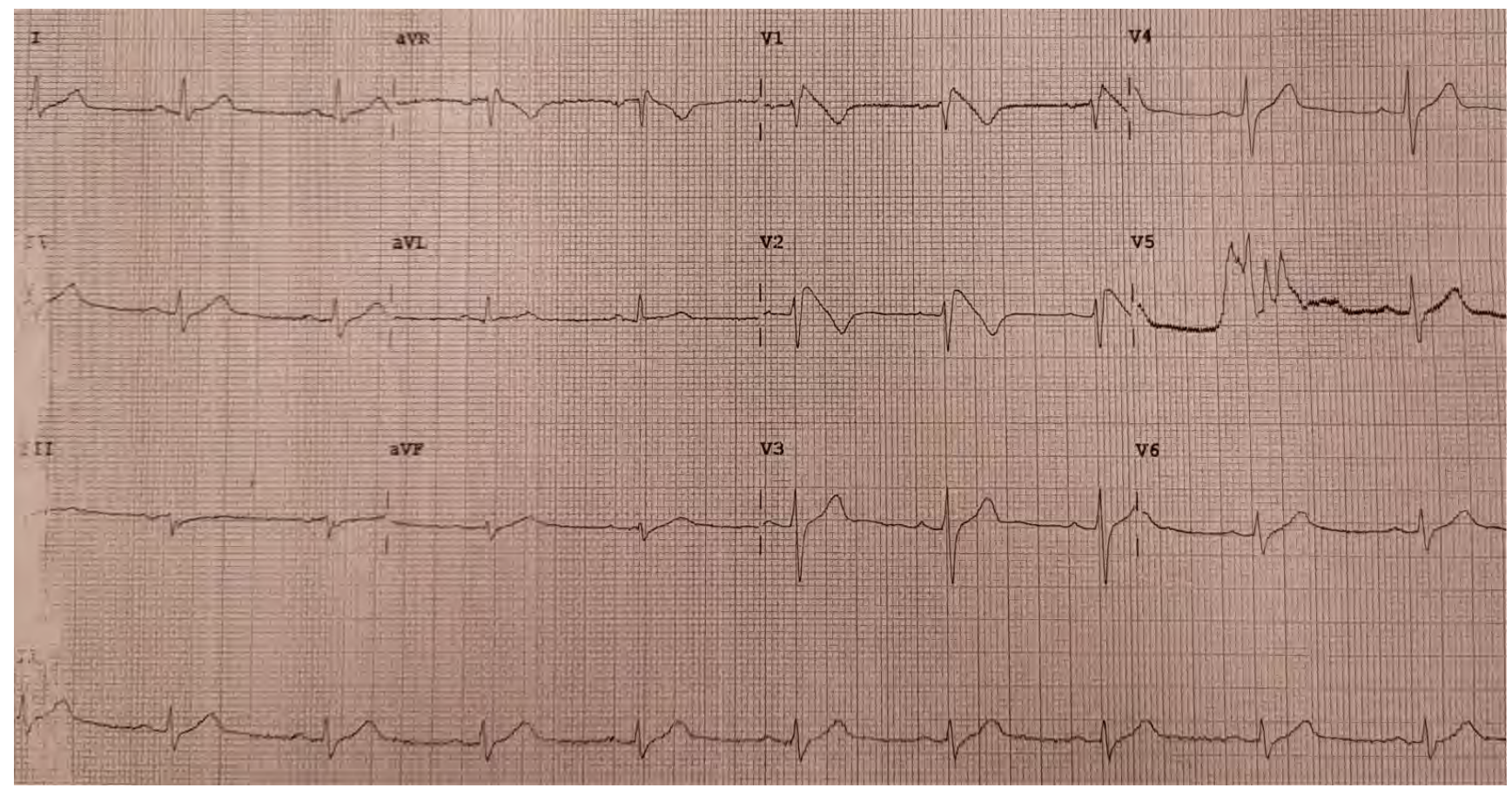

FIGURE 7A. ECG at presentation revealed Brugada type 1 pattern

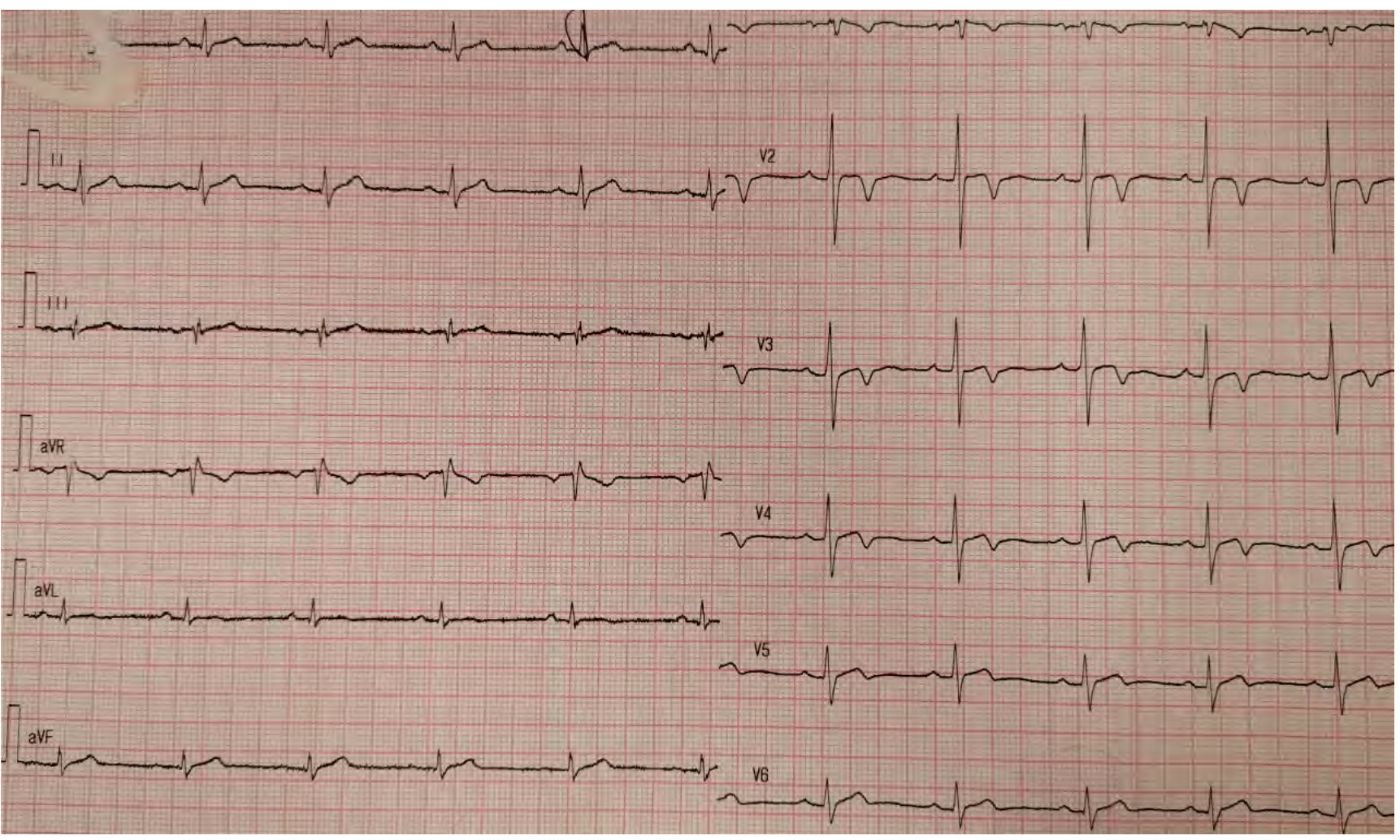

FIGURE 7B. After chest pain resolution, ECG showed type A Wellens pattern

appropriate management strategy, especially in high risk patients than can benefit from early invasive strategy.

The ECG remains the fastest, repeatable and most accesible instrument with diagnostic value, prognostic significance (risk stratification) and therapeutic implications (guiding the treatment strategy). The classification of ACS is based strictly on the ECG and considers the presence or absence of ST-segment elevation. However, there are forms of NSTEACS that behave and evolve as a STEMI. The particular ECG phenotypes considered as STEMI equivalents that we described prove the need to redefine the ECG signs of present or 
imminent coronary artery occlusion, especially LAD, because many patients may benefit from ear- ly invasive management instead of conservative pharmacological treatment.

\section{Conflict of interest: none declared} Financial support: none declared

\section{REFERENCES}

1. Benjamin EJ, Muntner P, Alonso A et al. Heart Disease and Stroke Statistics-2019 Update: A Report From the American Heart Association. Circulation. 2019 Mar 5;139(10):e56-e528.

2. Cervellin G, Rastelli G. The clinics of acute coronary syndrome. Ann Transl Med. 2016 May;4(10):191.

3. Braunwald E. Featuring: Eugene Braunwald. Eur Cardiol. 2019 Jul 11; 14(2): 130-133.

4. Ibanez B, James S, Agewall S et al. 2017 ESC Guidelines for the management of acute myocardial infarction in patients presenting with ST-segment elevation. Eur Heart J. 2018 Jan 7;39(2):119-177.

5. Thygesen K, Alpert JS, Jaffe AS et al. Third Universal Definition of Myocardial Infarction. Circulation. 2012 Oct 16;126(16):2020-2035.

6. Thygesen K, Alpert JS, Jaffe AS et al. Fourth universal definition of myocardial infarction (2018). Eur Heart J. 2019 Jan 14;40(3):237-269.

7. Shlipak MG, Lyons WL, Go AS et al. Should the electrocardiogram be used to guide therapy for patients with left bundle-branch block and suspected myocardial infarction? JAMA. 1999 Feb 24;281(8):714-9.

8. Sgarbossa EB, Pinski SL, Barbagelata A et al. Electrocardiographic diagnosis of evolving acute myocardial infarction in the presence of left bundlebranch block. GUSTO-1 (Global Utilization of Streptokinase and Tissue Plasminogen Activator for Occluded Coronary Arteries) Investigators. N Engl J Med. 1996 Feb 22;334(8):481-7.

9. Smith SW, Dodd KW, Henry TD et al. Diagnosis of ST-elevation myocardial infarction in the presence of left bundle branch block with the ST-elevation to S-wave ratio in a modified Sgarbossa rule. Ann Emerg Med. 2012 Dec;60(6):766-76.

10. Widimsky P, Rohác F, Stásek J et al. Primary angioplasty in acute myocardial infarction with right bundle branch block: should new onset right bundle branch block be added to future guidelines as an indication for reperfusion therapy? Eur Heart J. 2012 Jan;33(1):86-95.

11. Kurisu S, Inoue I, Kawagoe T et al. Electrocardiographic features in patients with acute myocardial infarction associated with left main coronary artery occlusion. Heart. 2004 Sep;90(9):1059-60.

12. Knotts RJ, Wilson JM, Kim E et al. Diffuse ST depression with ST elevation in aVR: Is this pattern specific for global ischemia due to left main coronary artery disease? J Electrocardiol. 2013 May-Jun; 46(3):240-8.

13. Oraii S, Maleki M, Tavakolian AA et al. Prevalence and outcome of STsegment elevation in posterior electrocardiographic leads during acute myocardial infarction. J Electrocardiol. $1999 \mathrm{Jul} ; 32(3): 275-8$.

14. Wung SF, Drew BJ. New electrocardiographic criteria for posterior wall acute myocardial ischemia validated by a percutaneous transluminal coronary angioplasty model of acute myocardial infarction. Am J Cardiol. 2001 Apr 15;87(8):970-4.

15. de Winter RJ, Verouden NJ, Wellens HJ et al. A new ECG sign of proximal LAD occlusion. N Engl J Med. 2008 Nov 6;359(19):2071-3.
16. Chioncel V, Avram A, lon AC et al. The de Winter T waves - an unusual presentation of left anterior descending artery occlusion. Romanian Journal of Cardiology. 2016; 26(3):330-3.

17. Dejanovic J, Stojsic-Milosavljevic A, Tranjkovic M et al. Atypical electrocardiographic presentations of myocardial infarction with ST elevation - ST elevation myocardial infarction equivalents. Med Pregl.

18. Verouden NJ, Koch KT, Peters RJ et al. Persistent precordial "hyperacute" T-waves signify proximal left anterior descending artery occlusion. Heart. 2009 Oct;95(20):1701-6.

19. Gerson MC, Phillips JF, Morris SN et al. Exercise-induced U-wave inversion as a marker of stenosis of the left anterior descending coronary artery. Circulation. 1979;60:1014-1020.

20. Gerson MC, McHenry PL. Resting U wave inversion as a marker of stenosis of the left anterior descending coronary artery. Am J Med. 1980;69:545-550.

21. de Zwaan C, Bär FW, Wellens HJ. Characteristic electrocardiographic pattern indicating a critical stenosis high in left anterior descending coronary artery in patients admitted because of impending myocardial infarction. Am Heart J. 1982 Apr;103(4 Pt 2):730-6.

22. Nisbet B, Zlupko G. Repeat Wellens' syndrome: Case report of critical proximal left anterior descending artery restenosis. J Emerg Med. 2010 Sep;39(3):305-8.

23. Rhinehardt J, Brady WJ, Perron AD et al. Electrocardiographic manifestations of Wellens' syndrome. Am J Emerg Med. 2002 Nov; 20(7):638-43.

24. Kobayashi A, Misumida N, Aoi S et al. Prevalence and Clinical Implication of Wellens' Sign in Patients With Non-ST-Segment Elevation Myocardial Infarction Cardiol Res. 2019 Jun;10(3):135-141.

25. Tomé G, Freitas J. Induced Brugada syndrome: Possible sources of arrhythmogenesis. Rev Port Cardiol. 2017 Dec;36(12):945-956.

26. Antzelevitch C, Yan GX, Ackerman MJ et al. J-Wave syndromes expert consensus conference report: emerging concepts and gaps in knowledge. Heart Rhythm. 2016 Oct;13(10):e295-324.

27. Wilde AA, Postema PG, Di Diego JM et al. The pathophysiological mechanism underlying Brugada syndrome: Depolarization versus repolarization. J Mol Cell Cardiol. 2010 Oct;49(4):543-53.

28. Baranchuk A, Nguyen T, Ryu MH et al. Brugada phenocopy: New terminology and proposed classification. Ann Noninvasive Electrocardiol. 2012 Oct;17(4):299-314.

29. Xu G, Gottschalk BH, Pérez-Riera A et al. Link between Brugada phenocopy and myocardial ischemia: Results from the International Registry on Brugada Phenocopy. Pacing Clin Electrophysiol. 2019 Jun;42(6):658-662. 2018 Jan; 71(7-8):241-246. 